\title{
Weighting Strategies for Combining Data from Dual-Frame Telephone Surveys: Emerging Evidence from Australia
}

\author{
Bernard Baffour ${ }^{1}$, Michele Haynes ${ }^{1}$, Mark Western ${ }^{1}$, Darren Pennay ${ }^{2,3}$, \\ Sebastian Misson ${ }^{3}$, and Arturo Martinez.
}

\begin{abstract}
Until quite recently, telephone surveys have typically relied on landline telephone numbers. However, with the increasing popularity and affordability of mobile phones, there has been a surge in households that do not have landline connections. Additionally, there has been a decline in the response rates and population coverage of landline telephone surveys, creating a challenge to collecting representative social data. Dual-frame telephone surveys that use both landline and mobile phone sampling frames can overcome the incompleteness of landline-only telephone sampling. However, surveying mobile phone users introduces new complexities in sampling, nonresponse measurement and statistical weighting. This article examines these issues and illustrates the consequences of failing to include mobile-phoneonly users in telephone surveys using data from Australia. Results show that there are significant differences in estimates of populations' characteristics when using information solely from the landline or mobile telephone sample. These biases in the population estimates are significantly reduced when data from the mobile and landline samples are combined and appropriate dual-frame survey estimators are used. The optimal choice of a dual-frame estimation strategy depends on the availability of good-quality information that can account for the differential patterns of nonresponse by frame.
\end{abstract}

Key words: Dual-frame telephone surveys; mobile phone sampling; nonresponse; weighting.

\section{Introduction}

The implementation of national social surveys is important for measuring social phenomena. In many countries, computer-assisted telephone interviewing (CATI) has become the most common mode for conducting such surveys, chiefly because of the relatively lower costs than face-to-face interviewing (Keeter et al. 2000; Keeter et al. 2006; Steeh 2008). However, telephone ownership is not universal and specific segments of the population, such as lower-income and ethnic-minority people, are at risk of being systematically excluded (Tucker et al. 2007; Brick et al. 2011; Busse and Fuchs 2012).

1 The University of Queensland - Institute for Social Science Research, Building 39A Campbell Road St Lucia, Brisbane, Queensland, 4067, Australia. Emails: b.baffour@uq.edu.au, m.haynes@uq.edu.au, m.western@uq.edu.au and amartinezjr@adb.org.

2 Australian National University - Australian Centre for Applied Social Research Methods, Canberra, Australian Capital Territory, Australia. Email: darren.pennay@srcentre.com.au

3 The Social Research Centre - Research Methodology, Melbourne, Victoria, Australia. Email: sebastian.misson@srcentre.com.au

Acknowledgments: We would like to thank the editor, associate editor and the three referees for their insightful comments and suggestions which considerably improved the article. This research was supported under Australian Research Council's Linkage Projects funding scheme (project number LP130100744 "Enhancing social research in Australia using dual-frame telephone surveys"). 
On average, current response rates of traditional landline telephone surveys have fallen to less than $60 \%$ for surveys conducted by national statistical institutes (Groves and Peytcheva 2008). For nongovernment surveys, response rates can be as low as ten percent (Pew Research 2012). These declines have implications for the representativeness of the sample with regard to the target population.

With mobile telephone use becoming increasingly prevalent in the population, including mobile telephone owners in the sampled population has the potential to address the coverage bias associated with traditional landline telephone surveys. This is because those who are more likely to be excluded from landline-based surveys often own a mobile telephone (Keeter et al. 2007; Pennay 2010; Brick 2011; Busse and Fuchs 2012). For instance, about $95 \%$ of adult Australians own a mobile telephone, compared to only $80 \%$ who own a landline (Australian Communications and Media Authority 2011). Additionally, there is an increasing trend for individuals to discard their landlines and rely solely on mobiles. In Australia, the proportion of adults who own a mobile telephone and live in a household without a landline telephone connection on which they receive calls has grown from five percent in 2005 to $29 \%$ in 2014 (Australian Communications and Media Authority 2015). This mobile-only population is excluded from surveys that rely solely on landline telephone sampling frames. Australian patterns mirror the experience in the United States, as shown in Figure 1.

Similar trends have been reported in Canada and in Europe (Brick et al. 2011; Mohorko et al. 2013). Exclusion of mobile-only individuals from social surveys has adverse consequences for survey estimates, as there are sociodemographic differences between individuals who own a mobile telephone and those who own a landline telephone (Brick et al. 2006 in the USA; Callegaro and Possio 2004 in Italy; Kuusela et al. 2008 in Finland; Vicente and Reis 2009 in Portugal; and Arcos et al. 2014 in Spain). Individuals living in mobile-only households are more likely to be younger, male, of a lower socioeconomic status, foreign-born, students, highly transient, in large cities, and in full-time employment (Blumberg and Luke 2014). These households are also more likely to experience poor health and adverse socioeconomic outcomes. (Barnes et al. 2015; Thomée et al. 2011;

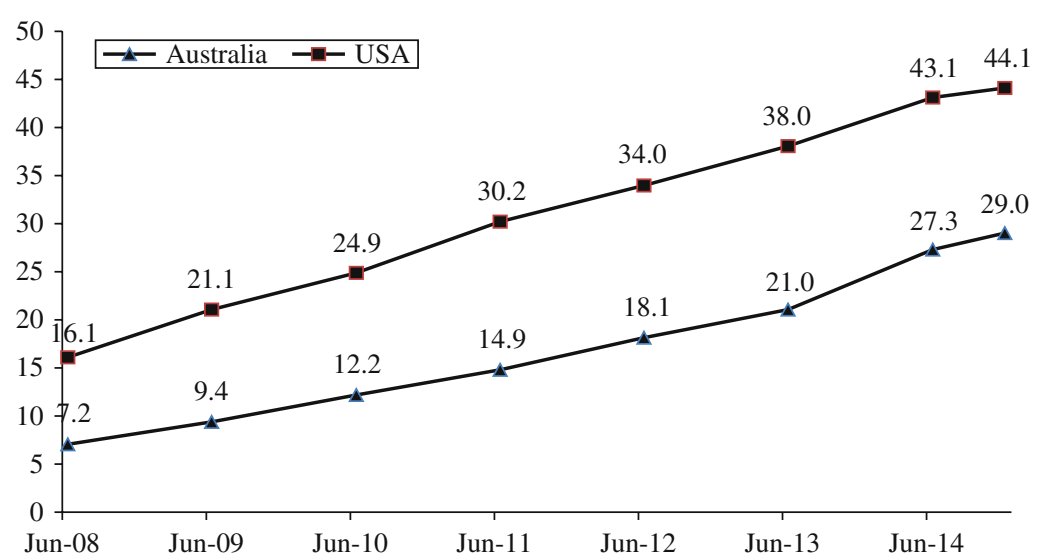

Fig. 1. The percentage of Australian and US adults with a mobile telephone and no fixed-line telephone service, June 2008 to June 2014. Sources: Australian data: ACMA (2015). USA data: Blumberg and Luke (2015). 
Hu et al. 2011). There are also differences between these groups in health and behavioural risk factors, such as smoking and alcohol use (Barr et al. 2012; Livingston et al. 2013). Altogether, this literature suggests that any survey that is directed at either landline-only or mobile-only individuals will suffer from coverage bias. To address this, an effective approach is to redesign traditional telephone surveys to include mobile telephones. Researchers agree that using dual-sampling frames will be an integral part of telephone surveys in the future (AAPOR 2010; Brick 2011).

The dual-frame telephone sampling approach involves supplementing telephone numbers from a randomly generated landline sampling frame with an independent sample of randomly generated mobile telephone numbers. This introduces additional complexity into the survey design and analysis. A number of estimators have been proposed to estimate population characteristics using data from dual-frame surveys. While each estimator has its advantages and limitations, the existing literature offers limited guidelines on choosing the appropriate estimator in a specific research context. Another unresolved issue is the extent to which nonresponse adjustments based on auxiliary data can be used to improve the efficiency of dual-frame estimation. Many of these methodological problems still remain in Australia, due to the lack of official statistics on mobile telephone usage and the fact that dual-frame telephone interviewing is a relatively recent innovation in comparison to other countries.

This article makes two contributions to the emerging literature on dual-frame surveys. First, it provides an up-to-date review of the available dual-frame estimators and their suitability to estimating population quantities. Second, it provides an empirical assessment of these estimators using nationally representative Australian data from dual-frame surveys. The findings presented in this article, while set within an Australian surveyresearch context, will be informative to researchers in other countries facing similar design decisions.

\section{Dual-Frame Sampling Theory}

\subsection{Background}

The objective of the dual-frame approach is to draw subpopulation samples from different sampling frames that, when combined, provide full coverage of the target population. The concept of dual-frame sampling dates back to the 1950s (Hartley 1962), but has not been applied to sampling from mobile and landline telephone frames until very recently (Lohr 2009; Arcos et al. 2014). Dual-frame surveys have become widely used by national statistical agencies, particularly for health surveys such as the US National Health Interview Survey (Blumberg and Luke 2007, 2014), and the Canadian Community Health Survey (Béland 2002). They often provide improved access to hard-to-reach populations (Kalton and Anderson 1986; Iachan and Dennis 1993; Flores Cervantes and Kalton 2008) and can reduce sampling costs by tailoring interview mode to respondent needs (Kennedy 2007; Lopez and Gonzalez-Barrera 2013).

Typically, telephone sampling frames will overlap, so that simply taking the union of all the frames will lead to duplication of the individuals in the population who appear in more than one frame. Duplication within combined sampling frames has posed a theoretical 
problem, with researchers interested in (1) the best way of combining the disparate information from the different frames, and (2) how to determine the reliability of the derived sample estimates (Fuller and Burmeister 1972; Hartley 1974; Bankier 1986; Skinner 1991). The problem of duplication is almost universal for dual-frame telephone surveys, as many people are likely to have access to both landline and mobile telephones.

\subsection{Estimation of Population Quantities from Combined Sampling Frames}

Figure 2 depicts the general situation when there are two sampling frames in telephone surveys (a landline telephone frame $\mathrm{L}$ and a mobile telephone frame $\mathrm{M}$ ), both with undercoverage of the target population, but when combined leading to improved population coverage. The frames $\mathrm{L}$ and $\mathrm{M}$ generate three mutually exclusive domains $-l$ (units in $\mathrm{L}$ alone), $m$ (units in $\mathrm{M}$ alone) and $l m$ (units in both $\mathrm{L}$ and $\mathrm{M}$ ). Following the classical texts of Hartley $(1962,1974)$ and Skinner (1991), Skinner and Rao (1996), and Lohr and Rao (2000, 2006), we denote the landline and mobile population sizes as $N_{L}$ and $N_{M}$, and the domain sizes as $N_{l}, N_{m}$, and $N_{l m}$, respectively. It follows that $N_{L}=N_{l}+N_{l m}$ and $N_{M}=N_{m}+N_{l m}$. Also, the total population size satisfies $N=N_{L}+N_{M}-N_{l m}=$ $N_{l}+N_{m}+N_{l m}$

Similarly, let $\mathrm{S}_{\mathrm{L}}$ and $\mathrm{S}_{\mathrm{M}}$ be samples, of size $n_{L}$ and $n_{M}$, drawn independently from the landline $\mathrm{L}$ and mobile $\mathrm{M}$ frames, respectively. Denote the overlapping sample as $\mathrm{S}_{l m}$, with sample size $n_{l m}$. Both the size of the overlapping population $N_{l m}$ and the size of the sample $n_{l m}$ are unknown. However, we do know that $n_{L}=n_{l}+n_{l m}^{L}$ and $n_{M}=n_{m}+n_{l m}^{M}$, where $n_{l m}^{L}$ is the overlap sample from the landline frame $\mathrm{L}$, and $n_{l m}^{M}$ is the overlap sample from the mobile telephone frame $\mathrm{M}$.

Finally, let $y_{i}$ denote the value associated with the observation for individual $i$, then the population statistic, given by $Y=\sum_{i=1}^{N} y_{i}$, is simply a sum of the units that appear in the domains $l, m$, and the overlap $l m$, that is landline only, mobile telephone only and both landline and mobile telephone users, respectively. Thus,

$$
Y=Y_{l}+Y_{m}+Y_{l m}=\sum_{i \epsilon l} y_{i}+\sum_{i \in m} y_{i}+\sum_{i \in l m} y_{i} .
$$

Suppose also that $y_{i}$ is observed for each individual in the samples $\mathrm{S}_{\mathrm{L}}$ and $\mathrm{S}_{\mathrm{M}}$, then the estimation problem is to use these data to construct a suitable estimator $\hat{Y}$ of $Y$. It is also of interest to find an estimator of the variance of $\hat{Y}$, denoted $\operatorname{var}(\hat{Y})$. Equation (1) shows how $Y$ can be computed using population data. Since the population universe, $\mathcal{U}$, can be decomposed into $l, m$ and $l m$, then, when we have a dual-frame sample, we can similarly

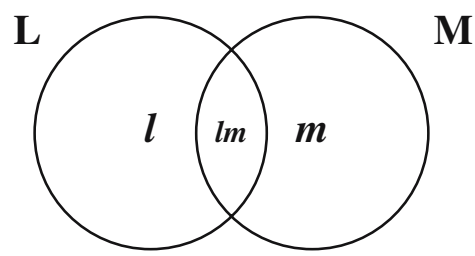

Fig. 2. Landline phone sampling frame $\mathrm{L}$ and mobile phone sampling frame $\mathrm{M}$ with overlap creating domains l, $m$ and $l m$. 
decompose the sample into the landline-only sample, the mobile-only sample and the dual sample, represented by $S_{l}, S_{m}$ and $S_{l m}$, respectively. It follows that the estimator for $Y$ using the sample information is now given by (2),

$$
\hat{Y}=\sum_{i \in S_{l}} w_{i} y_{i}+\sum_{i \in S_{m}} w_{i} y_{i}+\sum_{i \in S_{l m}} w_{i} y_{i}=\hat{Y}_{l}+\hat{Y}_{m}+\hat{Y}_{l m}
$$

where $w_{i}$ is the probability weight associated with unit $i$, and $\hat{Y}_{l}, \hat{Y}_{m}$, and $\hat{Y}_{l m}$ are sample statistics computed using information from the landline-only, mobile-only and dual samples.

The component $\hat{Y}_{l m}$ in Equation (2) can be estimated using either the data from individuals who reported having both landline and mobile phones from the landline sample, that is, $S_{l m}^{L}$, or the data from individuals who reported having both landline and mobile phones from the mobile sample, that is, $S_{l m}^{M}$. Although $S_{l m}^{L}$ and $S_{l m}^{M}$ are theoretically two independent samples from $S_{l m}$, it is convenient to think of both samples as duplicates from the same domain, and hence, the dual-frame estimators of the population quantity $Y$ can be calculated using a weighted estimate of the overlap according to frame $\mathrm{L}$ and frame $\mathrm{M}$,

$$
\hat{Y}_{l m}=\theta \hat{Y}_{l m}^{L}+(1-\theta) \hat{Y}_{l m}^{M}
$$

Combining (2) and (3) gives

$$
\hat{Y}=\hat{Y}_{l}+\hat{Y}_{m}+\theta \hat{Y}_{l m}^{L}+(1-\theta) \hat{Y}_{l m}^{M}
$$

where $\theta$ is referred to as the composite weight, $\hat{Y}_{l m}^{L}$ denotes the estimated statistic for individuals who use both landline and mobile phones derived from the landline sample, while $\hat{Y}_{l m}^{M}$ denotes the estimated statistic for sampled units who own both landline and mobile telephones derived from the mobile sample.

In general, methods to estimate $\hat{Y}$ from a dual-frame survey differ according to how the information from the individuals in the overlapping samples is used. Following Hartley (1962, 1974), Fuller and Burmeister (1972), Skinner and Rao (1996), and Lohr and Rao (2000), the choice of the composite weight $\theta$ is selected subject to some optimisation criteria which aim to minimise a loss function with respect to bias, variance or cost. Typically, $\theta$ is unknown and is replaced by $\hat{\theta}$, which is estimated from sample data (Skinner and Rao 1996), and the objective in dual-frame estimation is to find $\hat{\theta}$ such that estimators for the population quantity $Y$ are reliable and unbiased. Common approaches to combining the data from dual frames are summarised below.

\subsubsection{Screening Estimator}

The two extreme types of composite estimators for dual-frame surveys correspond to screening out sampled dual telephone users from either the landline or mobile telephone sample. In particular, a landline-only screener shown in (5) screens out those dual telephone users who were found in the landline sample. The mobile-only screener (6), on the other hand, screens out the mobile telephone sample dual users. This is accomplished by setting $\theta$ to be equal to 0 or 1 in (3), such that

$$
\text { when } \quad \theta=0, \quad \hat{Y}=\hat{Y}_{l}+\hat{Y}_{m}+\hat{Y}_{l m}^{M},
$$




$$
\text { and when } \theta=1, \quad \hat{Y}=\hat{Y}_{l}+\hat{Y}_{m}+\hat{Y}_{l m}^{L} \text {. }
$$

The screening approach is conceptually simple and estimation is straightforward. Note that (5) and (6) provide lower and upper bounds for other composite estimators.

\subsubsection{Average Estimator}

The average estimator, also known as the multiplicity estimator (Mecatti 2007) or fixedweight estimator (Hartley 1962), is the most commonly used estimator among the class of composite survey estimators proposed in the dual-frame sampling literature. Here, dual telephone users from the landline telephone sample contribute the same amount as the dual users from the mobile telephone sample in estimating the quantity of interest for all dual users. Thus, in this case $\theta=\frac{1}{2}$, and

$$
\hat{Y}_{\text {ave }}=\hat{Y}_{l}+\hat{Y}_{m}+\frac{1}{2} \hat{Y}_{l m}^{L}+\frac{1}{2} \hat{Y}_{l m}^{M} .
$$

There are several advantages to using this estimator. First, it is straightforward to compute and implement because the value of $\theta$ does not depend on the quantity of interest (Mecatti 2007). In the absence of nonresponse error, it can be shown that the multiplicity estimator is a consistent estimator of $Y$ (Bankier 1986). It is also straightforward to estimate the variance of $\hat{Y}_{\text {ave }}$, since $\theta$ is fixed across all individuals. However, the average estimator is not necessarily efficient and other estimators are often more statistically reliable because they use more information about the different frames.

\subsubsection{Hartley (Minimum-Variance) Estimator}

Although both the screening and average estimators are simple to compute, they are not necessarily as efficient as other estimators that incorporate information about how the data were collected under the different sampling frames. For instance, if the estimator $\hat{Y}_{l m}^{L}$ is more reliable than $\hat{Y}_{l m}^{M}$ in regards to estimating the overlapping domain quantity $Y_{l m}$, then it would make sense to place more weight on $\hat{Y}_{l m}^{L}$ than on $\hat{Y}_{l m}^{M}$. One way to achieve this is to minimise the variance of the target parameter $\hat{Y}$. Hartley $(1962,1974)$ showed that the variance of (4) is minimised when $\theta=\theta_{H}$,

$$
\theta_{H}=\frac{\operatorname{Var}\left(\hat{Y}_{l m}^{M}\right)+\operatorname{Cov}\left(\hat{Y}_{m}, \hat{Y}_{l m}^{M}\right)-\operatorname{Cov}\left(\hat{Y}_{l}, \hat{Y}_{l m}^{L}\right)}{\operatorname{Var}\left(\hat{Y}_{l m}^{L}\right)+\operatorname{Var}\left(\hat{Y}_{l m}^{M}\right)} .
$$

Thus, the Hartley estimator takes the form

$$
\hat{Y}_{H}=\hat{Y}_{l}+\hat{Y}_{m}+\hat{\theta}_{H} \hat{Y}_{l m}^{L}+\left(1-\hat{\theta}_{H}\right) \hat{Y}_{l m}^{M} .
$$

The advantage of the Hartley estimator is that it is asymptotically optimal among all fixedweight composite estimators. However, since the variance and covariance terms in (8) are unknown, the optimal value $\theta_{H}$ must be estimated from the data and different response variables will generate different values for $\hat{\theta}_{H}$, leading to internal inconsistency. For complex surveys, this inconsistency may be large (Lohr and Rao 2006). In addition, as the estimated variance and covariance terms depend on the quantity being estimated, the randomness of $\hat{\theta}_{H}$ should be taken into account when computing the variance of (9). 


\subsubsection{Fuller and Burmeister Estimator}

Fuller and Burmeister (1972) proposed modifying the Hartley estimator by making use of additional information about the overlapping units. The Fuller-Burmeister estimator is given by

$$
\hat{Y}_{F B}=\left(N_{L}-\hat{N}_{l m}\right) \hat{y}_{l}+\left(N_{M}-\hat{N}_{l m}\right) \hat{y}_{m}+\hat{N}_{l m}\left(\hat{\theta}_{F B} \hat{y}_{l m}^{L}+\left(1-\hat{\theta}_{F B}\right) \hat{y}_{l m}^{M}\right)
$$

where $\hat{y}_{l}, \hat{y}_{m}, \hat{y}_{l m}^{L}$, and $\hat{y}_{l m}^{M}$ are the sample estimates from the landline-only, mobile-only, dual users (from landline), and dual users (from mobile) samples. Finally, $\hat{N}_{l m}$ is the smallest root of the quadratic equation

$$
\left(n_{L}+n_{M}\right) x^{2}-\left(n_{L} N_{M}+n_{M} N_{L}+n_{l m}^{L} N_{L}+n_{l m}^{M} N_{M}\right) x+\left(n_{l m}^{L}+n_{l m}^{M}\right) N_{L} N_{M}=0 .
$$

The Fuller-Burmeister weights differ for different response variables and are therefore not internally consistent. Nevertheless, it can be shown that the Fuller-Burmeister estimator is more efficient than the Hartley estimator (Skinner and Rao 1996). Skinner and Rao (1996) proposed modifying this estimator by accounting for the complex sampling design through pseudomaximum-likelihood estimation, and showed that the weight adjustments do not depend on the covariances of the particular response being studied.

\subsubsection{Pseudomaximum-Likelihood Estimator (Skinner and Rao Estimator)}

In complex surveys, maximum-likelihood estimators do not usually have closed analytic forms (Gong and Samaniego 1981). To provide an internally consistent composite estimator for dual-frame surveys, Skinner and Rao (1996) proposed a pseudomaximumlikelihood estimator that uses a fixed value $\theta_{P M L}$ for any population characteristic of interest. The proposed approach draws strongly on the ideas of Fuller and Burmeister (1972) but aims to find a consistent dual-frame estimator through pseudolikelihood maximisation. Therefore, in order to obtain the pseudomaximum-likelihood estimator of the (unknown) population quantity, that is population total, average or proportion, first define $\hat{N}_{l m}^{L}=\frac{N_{L}}{n_{L}} n_{l m}^{L}$ and $\hat{N}_{l m}^{M}=\frac{N_{M}}{n_{M}} n_{l m}^{M}$. The pseudomaximum-likelihood estimator is given by

$$
\begin{aligned}
\hat{Y}_{P M L}= & \left(N_{L}-\hat{N}_{l m}^{P M L}(\theta)\right) \hat{y}_{l}+\left(N_{M}-\hat{N}_{l m}^{P M L}(\theta)\right) \hat{y}_{m} \\
& +\hat{N}_{l m}^{P M L}(\theta)\left\{\theta \hat{y}_{l m}^{L}+(1-\theta) \hat{y}_{l m}^{M}\right\}
\end{aligned}
$$

where $\hat{N}_{l m}^{P M L}(\theta)$ is a function of $\hat{N}_{l m}^{L}, \hat{N}_{l m}^{M}$ and $\theta=\theta_{P M L}$ which is the smaller of the roots of the quadratic equation

$$
\left[\frac{\theta}{N_{M}}+\frac{1-\theta}{N_{L}}\right] x^{2}-\left[1+\theta \frac{\hat{N}_{l m}^{L}}{N_{M}}+(1-\theta) \frac{\hat{N}_{l m}^{M}}{N_{L}}\right] x+\theta \hat{N}_{l m}^{L}+(1-\theta) \hat{N}_{l m}^{M}=0 .
$$

Skinner and Rao (1996) showed that the asymptotic variance of $\hat{N}_{l m}^{P M L}(\theta)$ is minimised when

$$
\theta_{P}=\frac{N_{l} N_{M} \operatorname{Var}\left(\hat{N}_{l m}^{L}\right)}{N_{l} N_{M} \operatorname{Var}\left(\hat{N}_{l m}^{L}\right)+N_{m} N_{L} \operatorname{Var}\left(\hat{N}_{l m}^{M}\right)} .
$$


In practice, $N_{l}, N_{m}$ and the variances are not known and so are estimated from the data, resulting in

$$
\hat{\theta}_{P}=\frac{\hat{N}_{l} N_{M} \widehat{\operatorname{Var}}\left(\hat{N}_{l m}^{L}\right)}{\hat{N}_{l} N_{M} \widehat{\operatorname{Var}}\left(\hat{N}_{l m}^{L}\right)+\hat{N}_{m} N_{L} \widehat{\operatorname{Var}}\left(\hat{N}_{l m}^{M}\right)}
$$

where $\hat{N}_{l m}^{P M L}(\theta)$ is the Fuller-Burmeister estimate of the overlapping population, and $\hat{N}_{l}=N_{L}-\hat{N}_{l m}^{P M L}(\theta) \approx N_{L}-\hat{N}_{l m}^{L}$ and $\hat{N}_{m}=N_{M}-\hat{N}_{l m}^{P M L}(\theta) \approx N_{L}-\hat{N}_{l m}^{M}$.

The pseudomaximum-likelihood estimator avoids the internal consistency problems present in the Hartley and Fuller-Burmeister estimators and has smaller mean squared error even in the presence of domain misclassification (Lohr and Rao 2000, 2006).

\subsubsection{Single-Frame Estimator}

The estimators discussed in Subsubsections 2.2.1 - 2.2.5 are based on choosing the composite weight subject to an optimisation criterion, for example minimising the variance in the class of linear unbiased estimators. The single-frame estimator assumes just one frame that encompasses information about both mobile telephone and landline use for each individual. Unlike the previous composite estimators that entail two-stage estimation of the population quantity, the single-frame estimator has an implicit adjustment in the estimation of the survey weights (i.e., inverse of selection probabilities) for sampled units from different frames. Assuming that landline and mobile telephone samples were drawn independently, the inclusion probability for the $i$ th sampled individual is given by $\pi_{i}^{L}+\pi_{i}^{M}-\pi_{i}^{L} \pi_{i}^{M}$; thus, $w_{i}=\frac{1}{\pi_{i}^{L}+\pi_{i}^{M}-\pi_{i}^{L} \pi_{i}^{M}}$.

The single-frame estimator uses the same set of weights for all response variables, and is therefore internally consistent (for details, see Bankier 1986). However, calculating the weights in the overlapping domain requires knowledge of the inclusion probability of each unit for both frames. The single-frame estimator is always less efficient than the Hartley estimator (Skinner and Rao 1996).

\subsubsection{Adjusting for Differential Nonresponse from Dual Frames}

Accounting for the multiple-frame coverage is one stage of the weighting adjustment, but this does not result in unbiased population estimates in most cases (Brick et al. 2011). Despite the best efforts, in every survey nonresponse occurs and is differential by social, economic, demographic, and geographical characteristics. Therefore, a second weighting adjustment is required to ensure that the weighted sample is representative of the population. This is achieved through calibrating the estimates to be consistent with known population benchmarks through poststratification (Holt and Smith 1979; Little 1993). While there are a number of different methods of poststratification, we will be using poststratification raking to repeatedly adjust the sample margins to the corresponding population control marginal totals. By using auxiliary information, we can ensure that the sample aligns to the population benchmarks for a set of characteristics. In Australia, raking is used in most official surveys to adjust for the effect of nonresponse (ABS 2014).

The poststrata (or population benchmarks) used for the raking are location (State capital, Rest of State), age group (18-24, 25-39, 40-49, 50-64, 65+), sex (Male, Female), 
educational attainment (University Graduate vs Non-University Graduate), birth place (Australian, Non-Australian), and telephone status (Mobile only, Dual user, Landline only). Aggregate data available on these population characteristics were available from the 2011 national census (Australian Bureau of Statistics 2012). The raking was then accomplished through adjusting the sample counts in each of these poststrata to the known population marginal control totals through an iterative proportional fitting procedure. This essentially combines the data from the survey with the aggregate information on the population from sources that have greater precision and unbiasedness (for instance the census) to adjust for nonresponse bias.

An important caveat is that these adjustments for nonresponse are based on associations of social, demographic, and selected characteristics with a known model of the probability to respond to surveys (Brick 2013). Moreover, reliable external information is required to benchmark the sample characteristics to these known population characteristics. Evidence from the USA shows that it is necessary to adjust for differential nonresponse bias by telephone type and usage (Brick et al. 2006; Brick et al. 2011).

It has been demonstrated that the different sampling frames have different response profiles, and hence population-level information about the profiles of coverage on the landline and mobile frames will be important for the poststratification. Unfortunately, in Australia, as in most other countries, detailed demographic information by telephoneusage status is not available. However, we can estimate the proportion of the population by telephone status broadly into landline only, dual landline and mobile, and mobile only. This information on telephone-usage status from the national media regulatory body, in addition to population figures from the Census of Population and Housing, will be used in the nonresponse adjustment.

\section{Dual-Frame Telephone Sampling in Australia}

\subsection{The Omnibus Surveys}

\subsubsection{Overview}

Compared to the USA, the Australian dual-frame experience is relatively new, with the earliest survey conducted in 2010 . That survey showed that $72 \%$ of respondents from the landline frame and $78 \%$ from the mobile frame used both types of telephone. The analysis of the survey data also found significant differences between mobile and landline telephone users. In particular, mobile users were more likely to be younger, reside in a capital city, be born outside Australia, and to be studying and living in group households (Pennay 2010). A larger omnibus survey using random digit dialling (RDD) that helped generate landline and mobile telephone sampling frames was administered in January 2012. This (first dual-frame omnibus survey) was designed to provide nationally representative statistics. The landline sample was proportionally stratified by geographical location across Australia. Selection probabilities for the landline sampling frame were derived through size quotas for capital city and noncapital city regions of the Australian states/territories. As there were no geographic identifiers available to stratify the mobile telephone sampling frame in Australia, a simple national random sampling frame was 
devised. Data collection was via computer-assisted telephone interviewing (CATI) and the in-scope population for the dual-frame survey was Australian residents aged 18 years and over, who are contactable by either a landline or mobile telephone (see Appendix A for questionnaire items on phone status). A subsequent omnibus survey with a very similar design and execution was conducted in March 2013 to further explore emerging trends describing mobile-telephone-only individuals in Australia (see Pennay and Vickers 2012, 2013).

\subsubsection{Survey Procedures, Call Results, and Analysis of Response}

The analysis presented in this article is based on the data from the 2012 survey. This survey was chosen due to its temporal proximity to the 2011 Australian Census of Population and Housing - the source of many of our external benchmarks. The 2012 survey comprised 1,012 interviews completed via the landline sample frame with a response rate (AAPOR Response Rate 3, as defined in AAPOR 2011) of 22.2\%, while the mobile sample frame yielded 1,002 completed interviews with a response rate of $12.7 \%$. The average interview length was 19.8 minutes for both samples. A total of 76,342 calls were placed to achieve the total 2,014 completed interviews, which equates to an interview every 37.9 calls, but this average number differed significantly $(p<0.001)$ by frame with 27.7 for the landline frame and $\mathbf{4 8 . 2}$ for the mobile frame. The main reason for this was that roughly a third $(32.5 \%)$ of all calls to mobile telephones resulted in voicemail outcomes, compared with $14.3 \%$ for landlines.

A number of strategies were used to maximise response and participation, including repeated callbacks to establish contact, the operation of a 1800 (free-to-call) number by the survey organisation, and leaving messages on answering machines/voicemail. Additionally, refusal conversion interviewing was used to identify the reasons for refusal and discretionary calls made to those identified as 'soft refusals', and the survey offered interviewing in languages other than English. Finally, an unlimited call cycle was used for the survey. This had the advantage of enabling interviews to be achieved with hard-toreach individuals (a six-call cycle is typical), and ten percent (197 interviews) were achieved from the seventh or subsequent call attempt.

In terms of final call outcomes, there was a much higher proportion of telephone answering devices (answering machines/voicemail) for the mobile frame $(20.0 \%)$ compared to the landline frame $(7.4 \%)$. There was also a higher proportion of 'no answer' outcomes among the mobile frame $(24.3 \%)$ compared with the landline frame $(12.9 \%)$. There was a higher proportion of nonworking or disconnected numbers in the mobile frame $(17.9 \%)$ compared with the landline frame $(6.6 \%)$. The relatively high number of uncontactable numbers in the mobile frame is reflected in the much higher ratio of records used per interview in the mobile frame (11.6:1), compared with the landline frame (6.1:1).

The results, in Table 1, show differences in the age profiles and country of birth profiles for respondents in the two samples (with higher proportions of younger and overseas-born people included in the mobile sample). Since the mobile telephone interviews were conducted with little control over the geographical distribution since location information is not available, more interviews were conducted in the more populated regions. The mobile telephone sample contained a larger proportion of males, a larger proportion of those residing in rented group households, and were predominantly in the capital cities. Furthermore, the mobile sample had a younger age profile and was more likely to be 


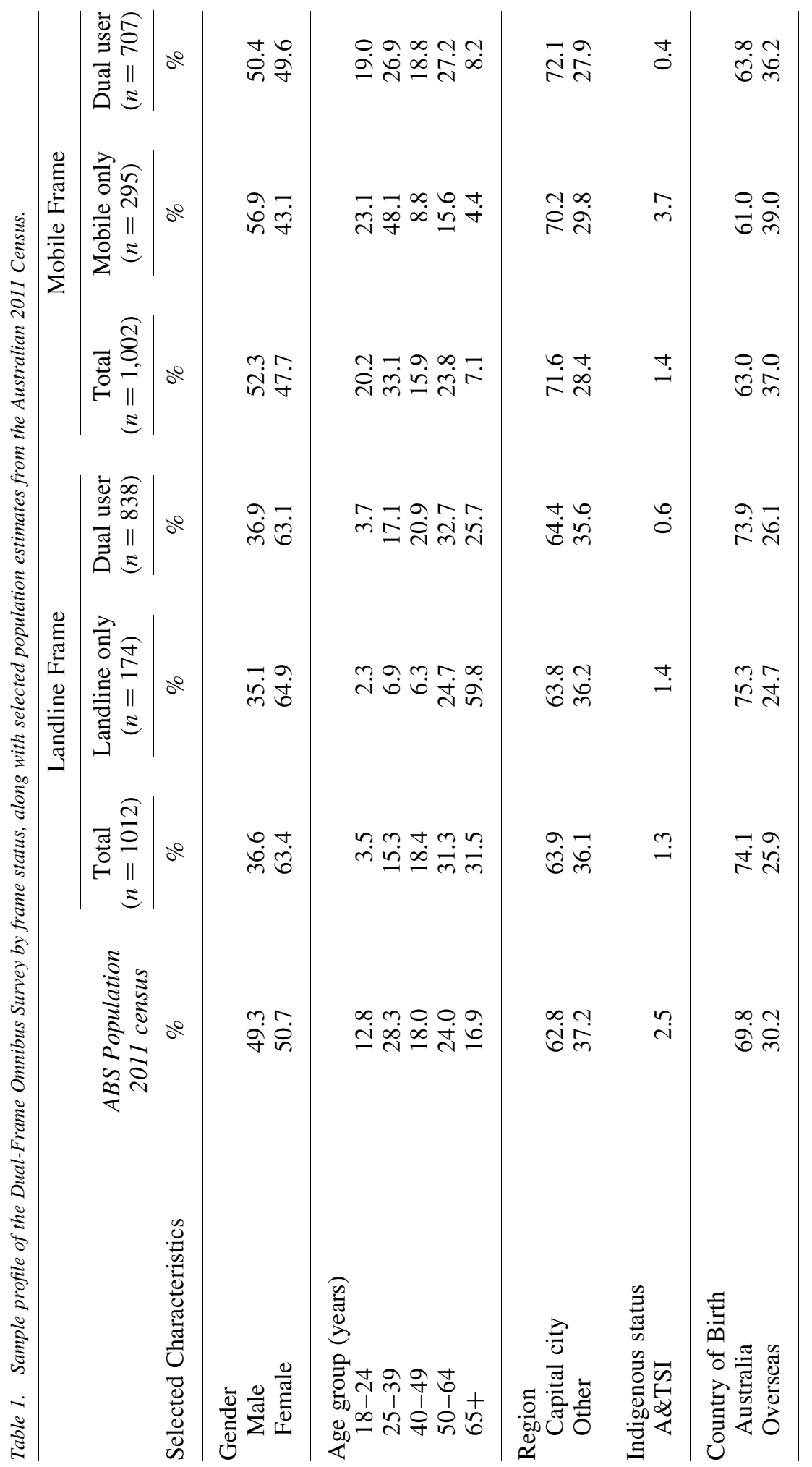




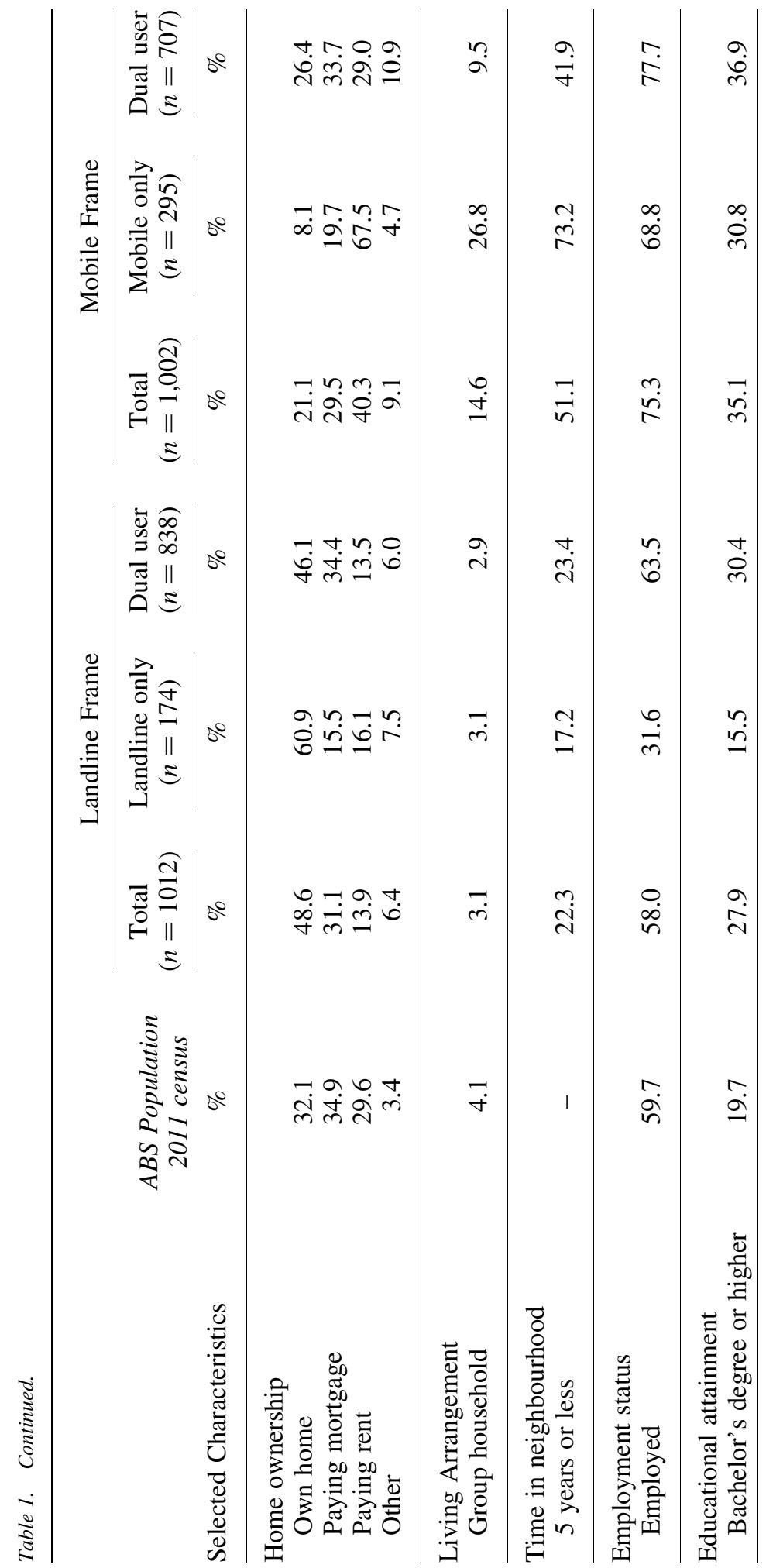


university educated and to be in employment. Additionally, the mobile telephone sample had a larger proportion of people born overseas in comparison with the landline sample. There were also differences in the characteristics of the dual telephone users depending on the sampling frame, with mobile-frame dual users more likely to be living in capital cities, group households and living in the neighbourhood for shorter periods, whereas landlineframe dual users were more likely to be Australian born and home owners. These differences have nontrivial implications for combining estimates from the landline and mobile frames.

\subsection{Variables}

A primary purpose of these surveys was to produce unbiased estimates for specific health characteristics and behaviours in the Australian population. The omnibus surveys collected data on health, attitudes, and behaviours, such as tobacco and alcohol consumption, experiences of discrimination, self-assessed medical health, and attitudes to the environment. In our evaluation of the performance of the various dual-frame estimators and weighting strategies, we considered a selection of the variables measuring these outcomes. Analyses of the data focused on:

1. investigating the link between transiency and telephone-usage status,

2. exploring the differences in social and health behaviour outcomes with telephoneusage status, and

3. examining the association between sedentary behaviour and telephone sampling.

To investigate the effect of transiency on responses from the landline and mobile telephone samples, we examined two variables measuring (i) group households and (ii) length of time in the neighbourhood (stayed less than five years or longer). We examined differences in social and health behaviour outcomes measured by smoking status, reports of being anxious or depressed, and belief in climate change. There is evidence to suggest a relationship between telephone-usage status and health-related behaviour. For example, it has been shown that the mobile-only population is likely to experience greater adverse health and behavioural outcomes (Blumberg and Luke 2014). Specifically, we considered smoking behaviour and incidence of depression and anxiety to investigate the hypothesis that the mobile-only population had poorer health outcomes (Lee et al. 2010; Thomée et al. 2011). In addition, we investigated attitudes to climate change, which are hypothesised to be related to age (Akter and Bennett 2011), with younger people more likely to believe that climate change is occurring and that humans are responsible for this. As mobile telephone usage is also associated with age, an estimate of the proportion of those who believed in climate change will be influenced by the choice of sampling frame and could be improved by combining data from both landline and mobile telephone sampling frames.

To investigate the association of telephone usage with sedentary behaviour, the recorded number of hours of television watched per day was analysed. According to the Australian Bureau of Statistics, the average amount of time spent watching television was just under three hours in 2008 (Australian Bureau of Statistics 2008). People who watch television for long periods of time are more likely to be those who are at home and this is related to accessibility by telephone. Long periods of television watching are also related 
to a sedentary lifestyle and poorer health outcomes (Hu et al. 2003), and combining data from both landline and mobile telephone sampling frames is likely to improve the accuracy of population estimates for time spent watching television.

Finally, to compensate for nonresponse in the sample and differences between telephone-usage status, we compute poststratification weights from a selection of variables that are often associated with nonresponse and survey quality: demographic (age and gender), socioeconomic (educational attainment), country of birth (comparing Australia born to overseas born), and geographic location (based on state of residence).

\section{Comparison of Dual-Frame Estimation Approaches}

In Section 2 we described different approaches to estimating a population quantity using combined data from dual overlapping sampling frames. In this section we assess the performance of these estimators empirically when applied to the 2012 Australian dualframe omnibus survey using information from the landline and mobile telephone samples. Three questions are addressed: Are there biases in the survey estimates if the mobile-only population is excluded? Are the biases in population estimates reduced when data from the two samples are combined? Finally, is there a preferred approach to weighting the combined samples?

\subsection{Weighting the Dual-Frame Omnibus Surveys for Multiple Coverage and Nonresponse}

To examine the biases in population estimates of the selected outcome variables, we firstly explored the differences in the response patterns of individuals by telephone sampling frame. In the presence of these differences, we then compared the population estimates using the various estimators described in Section 2 to combine sample data from the mobile telephone and landline frames. Lastly, as we have available information on age, sex, tenure status, and part-time employment from the most recent national census of Australia in 2011 (Australian Bureau of Statistics 2012), we compare these census estimates to estimates from the dual-frame survey to assess biases.

The five dual-frame estimators as described in Subsection 3.2 are computed for the analyses of the selected variables:

a. the screening estimators

b. the average estimator

c. the minimum-variance (Hartley) estimator

d. the pseudolikelihood (Skinner and Rao) estimator

e. the single-frame (Bankier) estimator.

The Skinner and Rao estimator (12) is similar to the Fuller and Burmeister estimator (10). But the Skinner and Rao estimator has the practical advantage of using the same weights for all variables and is approximately unbiased relative to the Fuller and Burmeister estimator. In addition, it is not possible to estimate the unknown population size $N$ using the Fuller-Burmeister estimator because the estimation process involves the inversion of a singular matrix (Skinner and Rao 1996; Lohr and Rao 2006). Thus, we do not consider it further. For the poststratified estimators, the weights are calibrated through 
a raking procedure so that the sample estimates correspond to known total population benchmarks across age, gender, educational attainment, country of birth, location, and telephone status. The survey estimates are examined before and after poststratification for the selected outcomes discussed in Subsection 3.3.

\subsection{Estimation of the Composite Weights Using Omnibus Survey Data}

The estimators identified above in (a) and (b) are straightforward to compute. For instance, the average estimator takes the simple average of the overlap between the two samples so that $\theta=1 / 2$. Additionally, the screening estimators are also easy to compute because $\theta$ is fixed to be either zero or one. For the other composite estimators, some algebra is required. As discussed in the previous section, estimation of $\theta$ is based on optimising some functions. In the case of Hartley's estimator, the optimal value of $\theta_{H}$ is computed by minimising the variance of the estimator. As shown in Equation (8), the optimal choice of $\theta_{H}$ is a function of the variances and covariances of the estimated domain totals, and the consequence of this is that they differ for each response variable. The optimal choice of the compositing weight was found to be 0.50 for having a bachelor's degree, 0.51 for being anxious or depressed, 0.46 for TV watching, 0.55 for daily smoking status, 0.34 for belief in climate change, 0.70 for group-household living arrangement, and 0.38 for short lengths of neighbourhood residence. This shows that there are differences in the choice of compositing value depending on the measure of interest ranging from 0.34 to 0.70 due to the associated variability in the different outcome measures.

The remaining estimators in (c), (d), and (e) are more complex. The pseudomaximumlikelihood estimator (15) depends on knowing the number of landline and mobile telephones in Australia, and then finding the maximum-likelihood estimator of the overlapping population, $\hat{N}_{l m}^{P M L}$ as the smallest root of (12). This is challenging as telephone-usage data is submitted by telecommunication service providers to the Australian Communications and Media Authority (ACMA), which is an independent statutory authority responsible for media regulation. In the USA, external information on telephone status is available through national surveys, such as the National Health Interview Survey (NHIS). This information allows for the adjustment of potential nonresponse biases associated with the different frames. Notably, there is area-level information available on mobile telephones in the USA. The situation is different in Australia. However, these figures from ACMA are the best available estimates of telephone coverage. We attempt to adjust for this lack of areal identifiers and any bias associated by including geographical information in the poststratification. Based on these figures, our computed estimate of the pseudomaximum-likelihood estimator is $\hat{\theta}_{P}=0.59$ (see Appendix B for details of how this was calculated). For the single-frame estimator, it is assumed that the landline and mobile samples are two stratified samples from the 'same' frame and are used to compute individual weights that have been adjusted for overlap in the two samples.

\subsection{Results}

Table 2 shows the population estimates for each measure, separately for the landline and mobile telephone samples and both with and without poststratification. Standard errors 


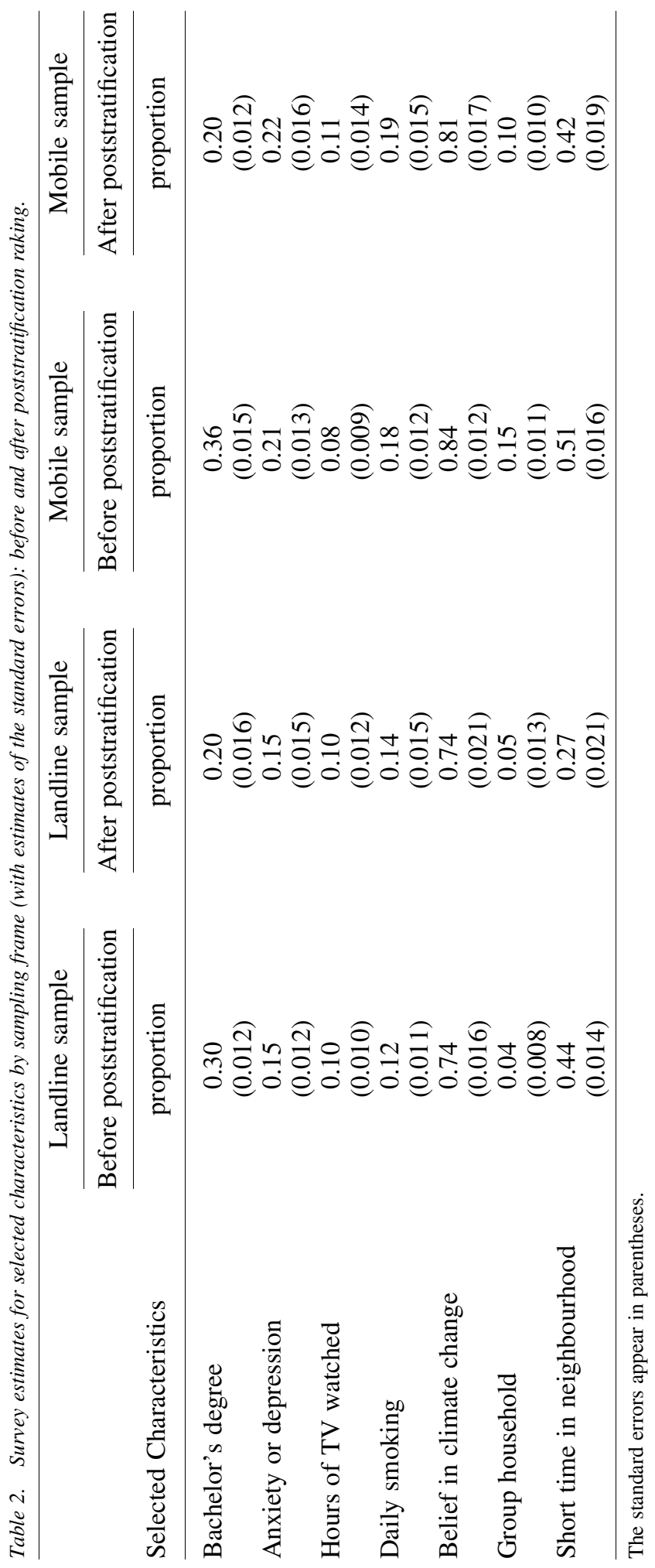


provide estimates of the precision, and these were computed using Taylor series approximation. However, qualitatively similar findings were observed when using the jackknife and bootstrap procedures (Lohr and Rao 2006; Lohr 2011). As previously shown, the estimates vary when using information from only the landline telephone frame or the mobile telephone frame. Since the estimators are estimated on independent samples, formal tests can be used to determine statistically significant differences. In particular, there are large and significant differences in the estimates for most of the variables analysed, including holding a bachelor's degree $(p=0.04)$, short length of time in neighbourhood $(p=0.002)$, having anxiety or depression $(p<0.001)$, smoking status $(p<0.001)$, living in a group household $(p<0.001)$, and long hours of TV watching $(p=0.085)$, before poststratification. This may be a consequence of differences in the individual profiles of mobile and landline phone users as well as differential nonresponse.

In general, the nonresponse-adjusted estimates should provide unbiased estimates of the population totals if we can construct a model that can completely explain the nonresponse mechanism. However, doing this usually changes the structure of the survey weights and can subsequently produce contrasting results for variables that are not included in the controls if these variables are related to the nonresponse mechanism (Deville and Särndal 1992). To demonstrate this, Table 2 shows the sample proportions for educational attainment measured by the qualification of having a bachelor's degree or not. The sample proportion of people with a bachelor's degree is higher before poststratification raking in both the landline telephone (29.7\%) and mobile telephone (35.7\%) samples. Approximately one third of respondents in the sample are educated to degree level and above, as compared to $20 \%$ in the Australian population, based on the 2011 Census. Thus raking has the effect of weighting the sample proportion to the national average of approximately $20 \%$. It is well known that people who are highly educated are more likely to respond to telephone surveys, and as such this population subgroup is overrepresented in both the landline and mobile telephone samples. Similarly, raking adjusts the estimates for under- and overrepresentation of the sample in characteristics measured by the other control variables.

Tables 3(a) and 3(b) provide a comparison of the different compositing approaches that integrate the information from the mobile and landline telephone samples, specifically addressing frame overlap. These compositing approaches apply weights to the dual users in the mobile and landline samples. We compare the two screening approaches (for landline and mobile telephones), the average, minimum-variance (Hartley), pseudolikelihood (Skinner and Rao), and single-frame estimators. In order to examine the effect of nonresponse we apply raking (to population control totals), and compare how the estimates from each of the estimators differ with and without raking. Table 3(a) shows the estimates without poststratification raking, while Table 3(b) shows the results with poststratification raking.

The screening estimators provide a lower and upper bound for the average, Hartley, pseudolikelihood and single-frame estimators. This is not surprising, because the screening estimators use $\theta=0$ or $\theta=1$ while the other composite estimators use $\theta$ values that fall within the $[0,1]$ range. In general, the choice of the compositing factor leads to differences in estimates of the population characteristics of interest. However, these differences are not statistically significant. The average estimator produced almost the same results to the 


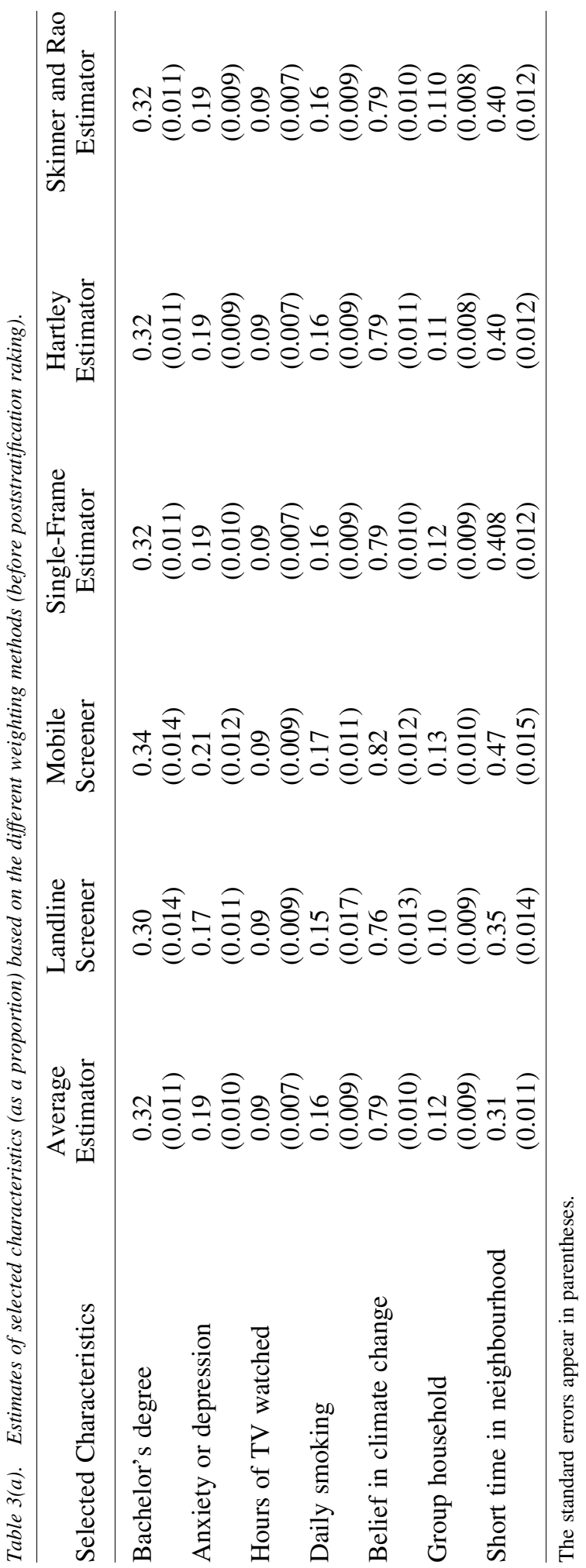




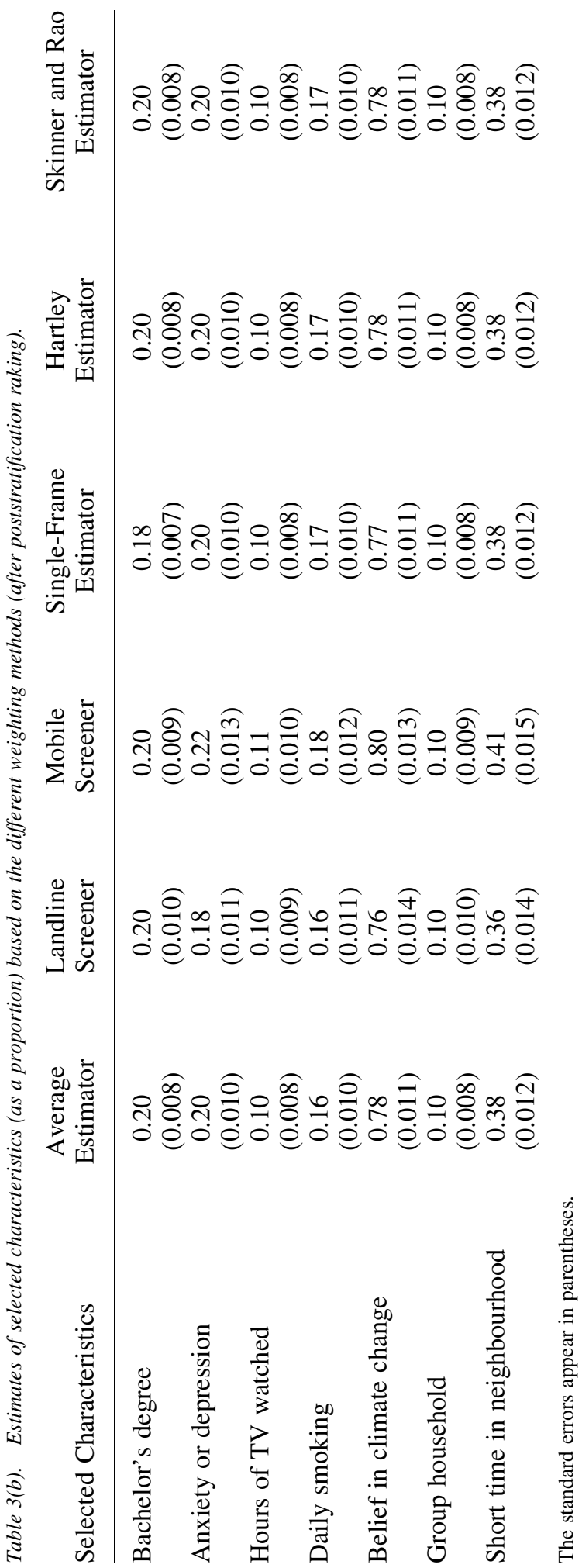


more complex compositing approaches, such as the pseudolikelihood and minimumvariance estimators. The results after the poststratification raking adjustment show that the average estimator, the Hartley estimator and the pseudomaximum-likelihood estimator all produce an equivalent estimate. The average estimator produced similar results to the more complex estimators. It is also worth noting that - with the exception of the variable time in neighbourhood - there is still little difference between the estimators, even before the raking to the benchmark control totals. Since the average estimator is theoretically inferior to the pseudomaximum likelihood, the consequence of this is that we may be lacking some control information for the benchmarking. In fact, the suggestion is that if population information on telephone status and the size of the mobile- and landline-frame populations was available (as it is in the USA and Europe), we would expect the average estimator to be radically inferior to the others.

These results are similar to the findings by Skinner and Rao (1996) and Lohr and Rao (2000). These authors have suggested that when there is little empirical evidence to select a preferred estimator, the decision to choose one estimator over the others should be made on theoretical and practical considerations, such as internal consistency and the availability of good-quality poststratification raking for benchmarking (Brick 2013; Arcos et al. 2014).

\subsection{Comparison of Composite Estimators With Census Figures}

To compare the performance of the dual-frame estimators with population quantities, we selected two variables for which existing census data was available, restricting the choice to those variables that were not used as benchmark controls (such as age, sex, country of birth, and region). Population estimates for part-time employment and housing-tenure status from the 2011 census were compared to estimates from the 2012 dual-frame omnibus survey. To ensure comparability to the survey, we restricted the census population to those aged 18 years and over.

We therefore examined how the estimates of part-time employment and tenure status varied first by doing the composite estimation (using the different estimators discussed) and then poststratifying to the control totals. We compared the precision and biases of the various weighting procedures, and the results are shown in Figure 3. If we have independent population information about the landline and mobile frames, such as demographic and geographical distributions, then this can be used to poststratify the mobile and landline samples to the control population controls. This is what is suggested by Brick et al. (2006) and Brick (2011). They suggest that by doing this, the fixed-weight average estimator is sufficient to reduce bias due to nonresponse, but they are dependent on knowing the response rates in the different domains. In Australia, currently, we do not have access to this level of telephone-usage population information, so it is of interest to see which estimator performs best.

Figure 3 shows estimates for part-time employment and tenure status (i.e., proportion of renters and home owners) using population census data, sample proportions and the dualframe estimators. To provide an indication of how the dual-frame estimators (average, single-frame, Hartley, and Skinner-Rao estimators) perform, panels (c) and (d) present the results before and after poststratification for these estimators. It may be due to these figures 
(a)

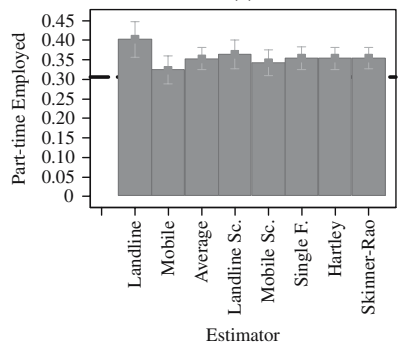

(b)

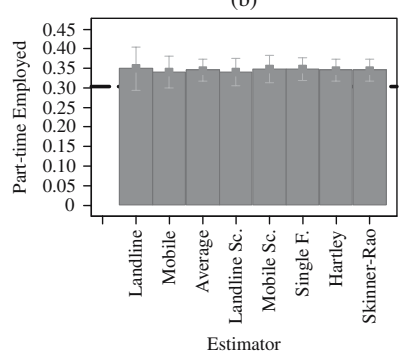

(c)

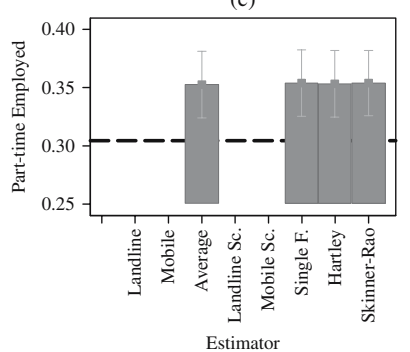

(d)

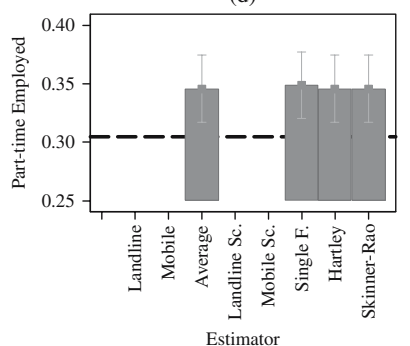

(a)

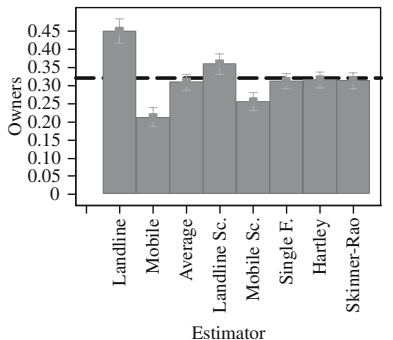

(b)

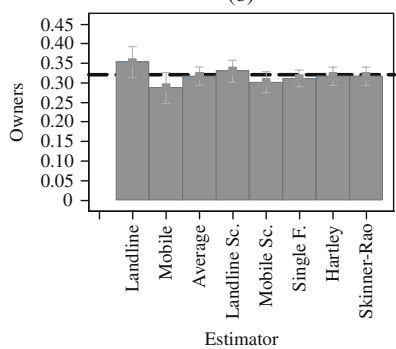

(c)

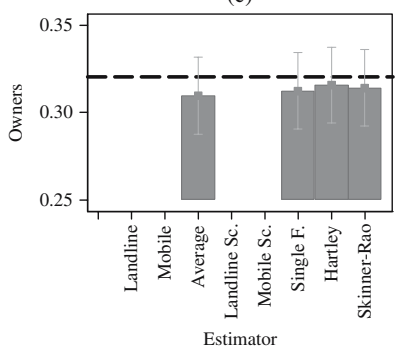

(d)

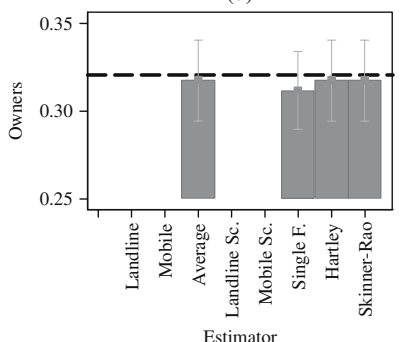

(a)

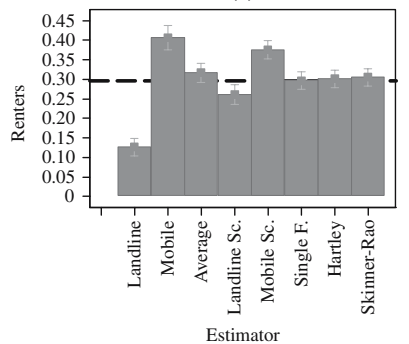

(b)

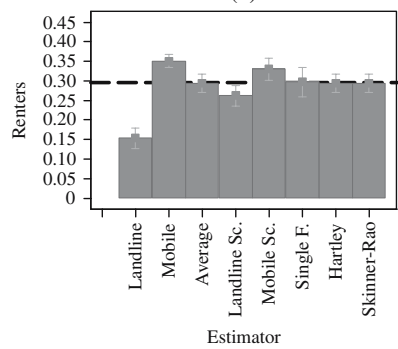

(c)

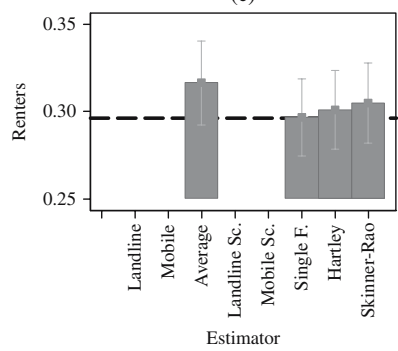

(d)

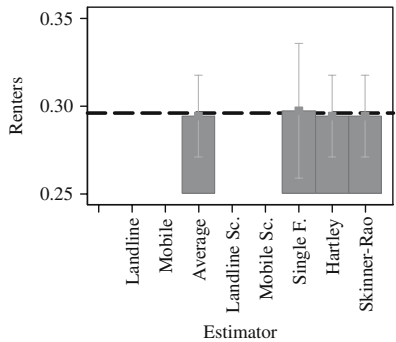

Fig. 3. Plots showing the estimates and the $95 \%$ confidence interval of selected characteristics for different dual-frame estimators; the left panel is for part-time employed, the middle panel is for owners, and the right panel is for renters. The census estimates are superimposed as the dotted line. For these plots (a) is the results before poststratification raking, $(b)$ is the results after poststratification raking; (c) gives the results of the four dualframe estimators (average, single-frame, Hartley and Skinner-Rao) before poststratification raking; (d) gives the results of the four dual-frame estimators (average, single-frame, Hartley and Skinner-Rao) after poststratification raking. 
that information from only the mobile or the landline telephone samples leads to biased estimates of the population quantities. The landline sample overestimates the proportion of home owners and underestimates the proportion of renters. It also underestimates those who are in part-time employment. The mobile sample produces a reasonable estimate of the proportion of part-time employees, but the estimates of home owners and renters are both biased by more than ten percent. These biases are still present, although to a lesser degree, after poststratification raking to adjust for noncoverage and nonresponse. The compositing weighting approaches overestimate the part-time employed; however, they are less biased in regards to the proportion of home owners or renters. This is true for all composite estimators apart from the screening approaches. This result is intuitive because, as shown above, the screeners tend to overestimate (or underestimate) and provide upper and lower bounds of the estimates.

After poststratification raking, the average, Hartley, and pseudolikelihood estimators give almost exactly the same results, and provide estimates that are closer to the population figures overall. Although the single frame does better than the screeners, it fares worse in comparison to the others. It appears to do worse if the poststratification raking is carried out before the combining. This supports Lohr (2011), who stated that the single-frame estimator may not be as efficient as the Skinner and Rao or the Hartley estimators, although there may be efficiency gains in raking to population totals. In the main, the choice of the estimator depends on the differential response patterns on the sampling frames, as well as the availability of auxiliary, good-quality data on the frame nonresponse patterns that can be used for benchmarking purposes (Kennedy 2007; Brick et al. 2011). Raking, in general, has the effect of reducing the bias since it calibrates the estimates to population totals. Raking after combining has the effect of preserving the structure of the estimates so as to be closer to the population quantities, even in the cases when the variables are not used directly in the poststratification raking.

Population benchmarks should be used to adjust the sample so that the weighted sample aligns to the population and produces unbiased estimates. But it is not entirely evident which population characteristics should be used as benchmarks. The general recommendation is to calibrate to age, sex, education, geography, race/ethnicity, marital status, home tenure, and population density, as well as telephone status (AAPOR 2010). In Australia, regular official statistics about the telephone status of the population are not available from the census or other government sources, meaning that the benchmark data available may not be of the desired accuracy. Owing to the uncertainty surrounding the available information on telephone status, we also carried out investigations as to how the population estimates changed when the raking was carried out with and without telephone status as control. The results did not show any differences (not shown here).

While it is preferable to adjust for both nonresponse and multiple coverage, and to seek to compensate for any biases associated with the differential patterns of coverage and response in the two sampling frames, the literature is not clear about how to do this. In our empirical study, we have found that the raking adjustment does appear to have the most influence. Similar results are found in US dual-frame studies (e.g., Brick et al. 2006; Brick et al. 2011). The main difference, however, is that the USA has good telephone-status information from external data. Nonetheless, in the absence of 'accurate' nationally representative data on telephone usage and availability, it is possible to rely on the age, 
sex, and other demographic and geographical characteristics that are related to telephone status. In our application to Australian dual-frame surveys, we have demonstrated that the uncertainty in the telephone-status information can be ameliorated by adjusting the samples to population characteristics from the census for differences in response profiles. As the mobile-only population increases, the situation will perhaps be different because of the potential nonsampling biases introduced.

\section{Conclusion}

Dual-frame telephone surveys will provide better coverage of the population than singleframe landline telephone surveys in most circumstances, due the absence of landline telephones in an increasing number of households and the exponential growth of households that are contactable only via mobile. We have shown that there are biases in survey estimates if the mobile-only population is excluded. These biases in the population estimates are significantly reduced when data from the mobile and landline sample are combined. However, although there are biases inherent in relying solely on a single frame, there are a number of issues that need to be addressed when proceeding to take a dual-frame survey approach. In the first instance, the combination of the information is not straightforward, as demonstrated by the various techniques available in the multiple-frame literature. Another aspect of estimation is the need to adjust for nonresponse through poststratification raking. Nonetheless, decisions about how to apply the poststratification raking are contingent on the availability of good-quality population-level information on sample characteristics that affect nonresponse and telephone status. As well as the current population benchmarks, the calculation of the nonresponse adjustments is contingent on phone-use benchmark information, which is routinely available in other countries, through nationally representative surveys that collect information on telephone status and usage. In Australia, there is no comparable survey that collects this type of information, and the influence this has on the computation of the compositing and nonresponse weights is unclear (Barr et al. 2014). This highlights the need for better population information on telephone usage in Australia, especially as the mobile-only population reaches the levels of the US.

Our results have demonstrated that the choice of an optimal dual-frame estimation approach depends on a number of factors. The first is the availability of good-quality information on telephone status. The second is the availability of raking information to account for the differential patterns of nonresponse. Essentially, our empirical results show that there is an interplay between the choice of dual-frame estimator and how to apply poststratification raking. In the absence of good-quality information on nonresponse, there is no preferable approach to weighting the combined samples. This supports Arcos et al. (2014), who showed that for the situation when accurate information on the mobile and landline populations is present, the single-frame and pseudomaximum-likelihood estimators give internally consistent results and are preferable. Although this was previously investigated by Lohr and Rao (2000, 2006), Brick et al. (2011) and Lohr (2011), we have now demonstrated this for the Australian dual-frame context. In particular, we have shown that the average estimator performs similarly to the more complicated estimators. We have shown that there are different conclusions as regards the best choice of dual-frame estimator because of the lack of independent information 
available on the landline- and mobile-frame population totals for compensating for nonresponse.

The need to properly understand the behaviour of the different compositing approaches in the presence of uncertain poststratification benchmarking totals is an area for future research, possibly through a rigorous Monte Carlo simulation study in which the dualframe estimators are compared across different features of the data. Due to the differential nonresponse that exists in the mobile and landline samples and the fact that - despite the best intentions of survey practitioners - there will be noncoverage in the samples, dualframe surveys need to adjust for nonresponse through poststratification. For this to work effectively, not only is information on the mobile and landline population totals needed, but detailed information on the differential nonresponse profiles of mobile and landline frames is also required.

\section{Appendix A - Questionnaire Items}

\section{Introductory Questions for Mobile Sample}

Intro1: Good morning/afternoon/evening. My name is $<$ SAY NAME $>$. I am calling from the Social Research Centre. The reason I'm calling is to see if you can help out with an important academic survey about health and wellbeing issues. To be eligible you need to be aged 18 years or over. The interview will take around 15 minutes depending on your answers. Would you be willing to do the survey at this time?

Intro2: May I just check whether or not it is safe to take this call at the moment. If not, I am happy to call you back when it is more convenient for you.

\section{Introductory Questions for Landline Sample}

Intro1: Good morning/afternoon/evening. My name is $<$ SAY NAME $>$. I am calling from the Social Research Centre. The reason I'm calling is to see if you can help out with an important academic survey about health and wellbeing issues. The results will be used to improve the quality of population research in Australia. The interview will take around 15 minutes depending on your answers. Would you be willing to do the survey at this time?

Intro2: For this research we'd like to speak to the person in the household aged 18 years and over who had the most recent birthday - will that be you? IF NECESSARY - This is just a way of randomising who we talk to in the household.

\section{Telephone Status Questions}

SMP1: To start with I have a question or two about your use of telephone services. Is there at least one working fixed line telephone inside your home that is used for making and receiving calls?

1. Yes

2. No

3. (Don't know)

4. (Refused) 


\section{*(IF LANDLINE SAMPLE OR SMP1 $=1$ (MOBILE SAMPLE WITH LANDLINE), CONTINUE, ELSE GO TO SMP3)}

SMP2: How many residential phone numbers do you have in your household not including lines dedicated to faxes, modems or business phone numbers? Do not include mobile phones.

INTEREVIEWER NOTE: If needed explain as how many individual landline numbers are there at your house that you use to make and receive calls?

1. Number of lines given (Specify ) RECORD WHOLE NUMBER (ALLOWABLE RANGE 1 TO 15)

2. No

3. (Don't know/Not stated)

SMP3: Do you have a working mobile phone?
1. Yes
2. No
3. (Don't know)
4. (Refused)

\section{Appendix B - Derivation of the Pseudomaximum-likelihood Estimator}

Recall that $n_{L}$ and $n_{M}$ are 1012 and 1002, and there are 838 landline dual users $\left(n_{l m}^{L}\right)$, and 707 mobile dual users $\left(n_{l m}^{M}\right) . N_{L}$ and $N_{M}$ are the population-level number of landlines and mobile phones in Australia (which is not known with accuracy). We therefore undertook a procedure to estimate these based on information provided by the Australian Bureau of Statistics (ABS) and the Australian Communications and Media Authority (ACMA). According to the ABS, there are 8,498,668 private dwellings in Australia, and ACMA estimates that $81 \%$ of people aged 18 years and over live in households with a landline connection (ACMA 2011, p.8). As households can have more than one landline connection we apply an adjustment factor of 1.05. This gives the estimated number of residential telephone numbers in Australia as 7228,117 $(=8,498,668 \times 0.81 \times 1.05)$.

Similarly, for the estimated number of people with mobile telephones, we first start with the proportion of the adult population with a mobile telephone in Australia as $89 \%$ (ACMA 2011, p. 13). There are 17,229,344 people aged 18 years and over according to the census. This gives the estimated number of mobile population as $15,334,107$ $((=0.89 \times 17,229,344)$.

Finally, landlines are household devices whereas mobiles are personal (individual) level, so to make it comparable we use the fact that there are 2.2 adults per household in Australia on average (Australian Bureau of Statistics 2012), and adjust the landline population from household level to individual level.

Since the population of dual users is not known, we use a result from Fuller and Burmeister (1972) to estimate dual users, such that $\hat{N}_{l m}^{L}=\frac{N_{L}}{n_{L}} n_{l m}^{L}=13,167,743.58$ for the landline dual-user population, and $\hat{N}_{l m}^{M}=\frac{N_{M}}{n_{M}} n_{l m}^{M}=10,819,574.50$ for the mobile dual-user population. 
The pseudomaximum-likelihood estimator of the dual-user population, $\hat{N}_{l m}^{P M L}$, is the smallest root of

$$
\left(n_{L}+n_{M}\right) x^{2}-\left(n_{L} N_{M}+n_{M} N_{L}+n_{l m}^{L} N_{L}+n_{l m}^{M} N_{M}\right) x+\left(n_{l m}^{L}+n_{l m}^{M}\right) N_{L} N_{M}=0 .
$$

After some algebra, this is found to be 11,900,766.15.

This estimate of the overlapping population seems reasonable and roughly equal to the average of the two estimates from the landline and mobile samples.

Finally to obtain the pseudomaximum-likelihood estimator of the compositing weight, we use the expression in Skinner and Rao (1996)

$$
\hat{\theta}_{P}=\frac{\hat{N}_{l} N_{M} \widehat{\operatorname{Var}}\left(\hat{N}_{l m}^{L}\right)}{\hat{N}_{l} N_{M} \widehat{\operatorname{Var}}\left(\hat{N}_{l m}^{L}\right)+\hat{N}_{m} N_{L} \widehat{\operatorname{Var}}\left(\hat{N}_{l m}^{M}\right)}
$$

where $\hat{N}_{l m}^{P M L}=11,900,766.15, \hat{N}_{l} \approx N_{L}-\hat{N}_{l m}^{P M L}$ and $\hat{N}_{m} \approx N_{L}-\hat{N}_{l m}^{P M L}$.

The pseudolikelihood-compositing weight $\theta_{P}$ is dependent on the variance terms $\operatorname{Var}\left(\hat{N}_{l m}^{L}\right)$ and $\operatorname{Var}\left(\hat{N}_{l m}^{M}\right)$, which are difficult to compute. However, we can use the fact that $\operatorname{Var}\left(\hat{N}_{l m}^{L}\right)=\left(\frac{N_{L}}{n_{L}}\right)^{2} \operatorname{Var}\left(n_{l m}^{L}\right)$ and $\operatorname{Var}\left(\hat{N}_{l m}^{M}\right)=\left(\frac{N_{M}}{n_{M}}\right)^{2} \operatorname{Var}\left(n_{l m}^{M}\right)$. However, it still remains to estimate $\operatorname{Var}\left(n_{l m}^{L}\right)$ and $\operatorname{Var}\left(n_{l m}^{M}\right)$, which are complex functions of the inclusion probabilities. Therefore, following on from Lohr and Rao (2000), $\widehat{\operatorname{Var}}\left(n_{l m}^{L}\right)$ and $\widehat{\operatorname{Var}}\left(n_{l m}^{M}\right)$ are estimated from the data. Substituting these values, the pseudomaximum-likelihood compositing weight is $\hat{\theta}_{P}=0.59$.

\section{References}

Akter, S. and J. Bennett. 2011. "Household Perceptions of Climate Change and Preferences for Mitigation Action: The Case of the Carbon Pollution Reduction Scheme in Australia." Climatic Change 109: 417-436. Doi: http://dx.doi.org/10.1007/ s10584-011-0034-8.

Arcos, A., M.M. Rueda, M. Trujillo, and D. Molina. 2014. "Review of Estimation Methods for Landline and Cell Phone Surveys." Sociological Methods and Research 44: 458-485. Doi: http://dx.doi.org/10.1177/0049124114546904.

American Association for Public Opinion Research (AAPOR). 2010. "Cell Phone Task Force Report. New Considerations for Survey Researchers When Planning and Conducting RDD Telephone Surveys in the US With Respondents Reached via Cell Phone Numbers." AAPOR. Available at: http://www.aapor.org/AAPORKentico/ Education-Resources/Reports/Cell-Phone-Task-Force-Report.aspx. (accessed 25 November 2015).

American Association for Public Opinion Research (AAPOR). 2011. "Standard Definitions: Final Disposition of Case Codes and Outcome Rates for Surveys 7th Edition." AAPOR. Available at: http://www.esomar.org/knowledge-and-standards/ research-resources/aapor-standard-definitions.php. (accessed 25 November 2015).

Australian Bureau of Statistics (ABS). 2008. "How Australians Use Their Time: Time spent on cultural activities. ABS cat.no.4153.0." Canberra: Australian Bureau of 
Statistics. Available at: http://www.abs.gov.au/ausstats/abs@.nsf/mf/4153.0 (accessed 25 November 2015).

Australian Bureau of Statistics (ABS). 2012. "Basic Community Profiles, Census 2011. Canberra: Australian Bureau of Statistics." Available at: http://www.abs.gov.au/ websitedbs/censushome.nsf/home/communityprofiles. (accessed 25 November 2015).

Australian Bureau of Statistics (ABS). 2014. "Australian Health Survey: Users' Guide, 2011-13." ABS cat.no. 4363.0.55.001. Canberra: Australian Bureau of Statistics. Available at: http://www.abs.gov.au/ausstats/abs@.nsf/mf/4363.0.55.001 (accessed 25 November 2015).

Australian Communications and Media Authority (ACMA). 2006, 2011, 2014, 2015. Communications Report, 2005-2006, 2010-2011, 2013-2014 \& 2014-2015 Series "Converging Communications Channels: Preferences and Behaviour of Australian Communications Users.” ACMA: Canberra. Available at: www.acma.gov.au/CommsReport. (accessed 25 November 2015).

Bankier, M.D. 1986. "Estimators Based on Several Stratified Samples With Applications to Multiple Finite Surveys." Journal of the American Statistical Association 81: 1074-1079. Doi: http://dx.doi.org/10.1080/01621459.1986.10478376.

Barnes, G.M., J.H. Hoffman, M.O. Tidwell, and J.W. Welte. 2015. "Gambling and Substance Use: Co-Occurrence Among Adults in a Recent General Population Study in the United States." International Gambling Studies 15: 55-71. Doi: http://dx.doi.org/ 10.1080/14459795.2014.990396.

Barr, M.L., J.J. van Ritten, D.G. Steel, and S.V. Thackway. 2012. "Inclusion of Mobile Phone Numbers Into an Ongoing Population Health Survey in New South Wales, Australia: Design, Methods, Call Outcomes, Costs and Sample Representativeness." BMC Medical Research Methodology 12: 177-185. Doi: http://dx.doi.org/10.1186/ 1471-2288-12-177.

Barr, M.L., R.A. Ferguson, P.J. Hughes, and D.G. Steel. 2014. "Developing a Weighting Strategy to Include Mobile Telephone Numbers Into an Ongoing Population Health Survey Using an Overlapping Dual Frame Design With Limited Benchmark Information.” BMC Medical Research Methodology 14: 102-112. Doi: http://dx.doi. org/10.1186/1471-2288-14-102.

Béland, Y. 2002. "Canadian Community Health Survey - Methodological Overview." Health Reports - Statistics Canada 13: 9-14.

Blumberg, S.J. and J.V. Luke. 2007. "Coverage Bias in Traditional Telephone Surveys of Low-Income and Young Adults.” Public Opinion Quarterly 71: 734-749. Doi: http:// dx.doi.org/10.1093/poq/nfm047.

Blumberg, S.J. and J.V. Luke. 2014. "Wireless Substitution: Early Release Estimates from the National Health Interview Survey, January-June 2014.” National Center for Health Statistics. Available at: http://www.cdc.gov/nchs/nhis/releases.htm (accessed 25 November 2015).

Blumberg, S.J. and J.V. Luke. 2015. "Wireless Substitution: Early Release Estimates from the National Health Interview Survey, July-December 2014.” National Center for Health Statistics. Available at: http://www.cdc.gov/nchs/nhis/releases.htm (accessed 25 November 2015). 
Brick, J.M. 2011. "The future of survey sampling." Public Opinion Quarterly 75: 872-888. Doi: http://dx.doi.org/10.1093/poq/nfr045.

Brick, J.M. 2013. "Unit Nonresponse and Weighting Adjustments: a Critical Review." Journal of Official Statistics 29: 329-353. Doi: http://dx.doi.org/10.2478/ jos-2013-0026.

Brick, J.M., I.F. Cervantes, S. Lee, and G. Norman. 2011. "Nonsampling Errors in Dual Frame Telephone Surveys." Survey Methodology 37: 1-12.

Brick, J.M., S. Dipko, S. Presser, and C. Tucker. 2006. "Nonresponse Bias in a Dual Frame Sample of Cell and Landline Numbers." Public Opinion Quarterly 70: 780-793. Doi: http://dx.doi.org/10.1093/poq/nfl031.

Busse, B. and M. Fuchs. 2012. "The Components of Landline Telephone Survey Coverage Bias. The Relative Importance of No-Phone and Mobile-Only Populations.” Quality \& Quantity 46: 1209-1225. Doi: http://dx.doi.org/10.1007/s11135-011-9431-3.

Callegaro, M. and T. Possio. 2004. "Mobile Telephone Growth and Coverage Error in Telephone Surveys." Polis: Research and Studies in Italian Society and Politics 18: 477-506.

Deville, J. and C.-E. Särndal. 1992. "Calibration Estimators in Survey Sampling.” Journal of the American Statistical Association 87: 376-382.

Flores Cervantes, I. and G. Kalton. 2008. "Methods for Sampling Rare Populations in Telephone Surveys." In Advances in Telephone Survey Methodology, edited by J.M. Lepkowski, C. Tucker, J.M. Brick, E.D. de Leeuw, L. Japec, P. Lavrakas, M.W. Link, and R.L. Sangster, 113-132. Hoboken, NJ: Wiley.

Fuller, W.A. and L.F. Burmeister. 1972. "Estimators for Samples Selected From Two Overlapping Frames." In Proceedings of the Social Statistics Section of American Statistical Association, August 14-17, 245-249. Alexandria, VA: American Statistical Association.

Gong, G. and F.J. Samaniego. 1981. "Pseudo Maximum Likelihood Estimation: Theory and Applications." The Annals of Statistics 9: 861 -869. Doi: http://dx.doi.org/10.1214/ aos/1176345526.

Groves, R.M. and E. Peytcheva. 2008. "The Impact of Non-Response Rates on NonResponse Bias.” Public Opinion Quarterly 72: 167-189. Doi: http://dx.doi.org/10. 1093/poq/nfn011.

Hartley, H.O. 1962. "Multiple Frame Surveys." In Proceedings of the Social Statistics Section of American Statistical Association, date of conference, 203-206. Alexandria, VA: American Statistical Association.

Hartley, H.O. 1974. "Multiple Frame Methodology and Selected Applications." Sankhya C 36: 99-118.

Holt, D. and T.M.F. Smith. 1979. "Post-Stratification." Journal of the Royal Statistical Society, Series A 142: 33-46.

Hu, S.S., L. Balluz, M.P. Battaglia, and M.R. Frankel. 2011. "Improving Public Health Surveillance Using a Dual Frame Survey of Landline and Cell Phone Numbers." American Journal of Epidemiology 173: 703-711. Doi: http://dx.doi.org/10.1093/aje/ kwq442.

Hu, F.B., T.Y. Li, G.A. Colditz, W.C. Willett, and J.E. Manson. 2003. "Television Watching and Other Sedentary Behaviours in Relation to Risk of Obesity and Type 2 
Diabetes Mellitus in Women." Journal of the American Medical Association (JAMA) 289: 1785-1791. Doi: http://dx.doi.org/10.1001/jama.289.14.1785.

Iachan, R. and M.L. Dennis. 1993. "A Multiple Frame Approach to Sampling the Homeless and Transient Population.” Journal of Official Statistics 9: 747-764.

Kalton, G. and D.W. Anderson. 1986. "Sampling Rare Populations.” Journal of the Royal Statistical Society, Series A 149: 65-82. Doi: http://dx.doi.org/10.2307/2981886.

Keeter, S., C. Miller, A. Kohut, R.M. Groves, and S. Presser. 2000. "Consequences of Reducing Non-Responses in a National Telephone Survey.” Public Opinion Quarterly 64: 125-148. Doi: http://dx.doi.org/10.1086/317759.

Keeter, S., C. Kennedy, A. Clark, T. Tompson, and M. Mokrzycki. 2007. "What's Missing From National Landline RDD surveys? The Impact of the Growing Cell-Only Population." Public Opinion Quarterly 71: 772-792. Doi: http://dx.doi.org/10.1093/poq/nfm053.

Keeter, S., S.C. Kennedy, M. Dimock, J. Best, and P. Craighill. 2006. "Gauging the Impact of Growing Non-Response on Estimates from a National RDD Telephone Survey." Public Opinion Quarterly 70: 759-779. Doi: http://dx.doi.org/10.1093/poq/nfl035.

Kennedy, C. 2007. "Evaluating the Effects of Screening for Telephone Service in Dual Frame RDD surveys.” Public Opinion Quarterly 71: 750-771. Doi: http://dx.doi.org/ $10.1093 / \mathrm{poq} / \mathrm{nfm} 050$.

Kuusela, V., M. Callegaro, and V. Vehovar. 2008. "The Influence of Mobile Telephones on Telephone Surveys.” In Advances in Telephone Survey Methodology, edited by J.M. Lepkowski, C. Tucker, J.M. Brick, E.D. de Leeuw, L. Japec, P.J. Lavrakas, M.W. Link, and R.L. Sangster, 87-112. Doi: http://dx.doi.org/10.1002/9780470173404.ch4.

Lee, S., J.M. Brick, E.R. Brown, and D. Grant. 2010. "Growing Cell-Phone Population and Noncoverage Bias in Traditional Random Digit Dial Telephone Health Surveys." Health Services Research 45: 1121-1139. Doi: http://dx.doi.org/10.1111/j.1475-6773. 2010.01120.x.

Little, R.J.A. 1993. "Post-Stratification: A Modeler's Perspective." Journal of the American Statistical Association 88: 1001-1012.

Livingston, M., P. Dietze, J. Ferris, D. Pennay, L. Hayes, and S. Lenton. 2013. "Surveying Alcohol and Other Drug Use Through Telephone Sampling: A Comparison of Landline and Mobile Phone Samples." BMC Medical Research Methodology 13: 41. Doi: http:// dx.doi.org/10.1186/1471-2288-13-41.

Lohr, S. 2009. "Multiple Frame Surveys.” In Handbook of Statistics, Sample Surveys: Design, Methods and Applications, edited by D. Pfeffermann and C.R. Rao, 71-88. Amsterdam: North Holland.

Lohr, S.L. 2011. “Alternative Survey Sample Designs: Sampling With Multiple Overlapping Frames.” Survey Methodology 37: 197-213.

Lohr, S. and J. Rao. 2000. "Inference From Dual Frame Surveys." Journal of the American Statistical Association 95: 271-280. Doi: http://dx.doi.org/10.1080/01621459.2000. 10473920.

Lohr, S. and J. Rao. 2006. "Estimation in Multiple-Frame Surveys." Journal of the American Statistical Association 101: 1019-1030. Doi: http://dx.doi.org10.1198/ 0162144506000000195.

Lopez, M.H. and A. Gonzalez-Barrera. 2013. "Inside the 2012 Latino Electorate." Washington, D.C.: Pew Research Center's Hispanic Trends Project. Available at: 
http://www.pewhispanic.org/2013/06/03/inside-the-2012-latino-electorate. (accessed 25 November 2015).

Mecatti, F. 2007. "A Single Frame Multiplicity Estimator for Multiple Frame Surveys." Survey Methodology 33: 151-157.

Mohorko, A., E. de Leeuw, and J. Hox. 2013. "Coverage Bias in European Telephone Surveys: Developments of Landline and Mobile Phone Coverage Across Countries and Over Time.” Survey Methods: Insights from the Field (SMIF) 2013, 1-13. Doi: http:// dx.doi.org/10.13094/SMIF-2013-00002.

Pennay, D.W. 2010. "Profiling the 'Mobile Only' Population. Results From a Dual-Frame Telephone Survey Using a Landline and Mobile Phone Sample Frame.” In Proceedings of the Australian Consortium for Social and Political Research Incorporated (ACSPRI) Social Science Methodology Conference, 1-3 December, Sydney, Australia. Available at: http://www.srcentre.com.au/docs/publications/dual_frame-survey_acspriconference-paper_finalv2.pdf?sfvrsn $=0$ (accessed 25 November 2015).

Pennay, D.W. and N. Vickers. 2012. "Dual-Frame Omnibus Survey." Technical and Methodological Summary Report. Social Research Centre Pty Ltd, Melbourne, Australia. Available at: http://www.srcentre.com.au/docs/event-workshop-july-2012/ dual-frame-omnibus-technical-report-(pennay).pdf?sfvrsn=2 (accessed 25 November 2015).

Pennay, D.W. and N. Vickers. 2013. "Second Dual-Frame Omnibus Survey." Technical and Methodological Summary Report. Social Research Centre Pty Ltd, Melbourne, Australia. Available at: http://www.srcentre.com.au/docs/publications/full-report-here. pdf?sfvrsn $=0$ (accessed 27 November 2015).

Pew Research Centre. 2012. "Assessing the Representativeness of Public Opinion Surveys.” Available at: http://www.people-press.org/2012/05/15/assessing-the-representativeness-of-public-opinion-surveys/ (accessed 25 November 2015).

Skinner, C.J. 1991. "On Efficiency of Raking Ratio Estimation for Multiple Frame Surveys.” Journal of the American Statistical Association 86: 779-784.

Skinner, C.J. and J.N.K. Rao. 1996. "Estimation in Dual Frame Surveys with Complex Designs." Journal of the American Statistical Association 91: 349-356.

Steeh, C. 2008. "Telephone Surveys.” In International Handbook of Survey Methodology, edited by E.D. de Leuuw, J.J. Hox, and D.A. Dillman, 221-238. New York: Routledge.

Thomée, S., A. Härenstam, and M. Hagberg. 2011. "Mobile Phone Use and Stress, Sleep Disturbances, and Symptoms of Depression Among Young Adults - A Prospective Cohort Study." BMC Public Health 11: 66. Doi: http://dx.doi.org/10.1186/ 1471-2458-11-66.

Tucker, C. and J.M. Brick. 2007. "Household Telephone Service and Usage Patterns in the United States in 2004: Implications for Telephone Samples." Public Opinion Quarterly 71: 3-22. Doi: http://dx.doi.org/10.1093/poq/nfl047.

Vicente, P. and E. Reis, E. 2009. The "Mobile Only Population in Portugal and its Impact in a Dual Frame Telephone Survey." Survey Research Methods 3: 105-111.

Received January 2015

Revised December 2015

Accepted January 2016 